\title{
MINIMIZATION PROBLEMS WITH LACK OF COMPACTNESS
}

\author{
MICHEL WILLEM \\ Institut de Mathématique Pure $\&$ Appliquée \\ Université Catholique de Louvain \\ Bâtiment Sc. I, Chemin du Cyclotrone 2 \\ B-1348 Louvain-la-Neuve, Belgium \\ E-mail: Willem@amm.ucl.ac.be
}

1. Introduction. A major progress in the calculus of variations since ten years is a systematic treatment of problems with lack of compactness. Our aim is to give an elementary approach to four typical cases. The methods are perhaps more important than the results. Lack of compactness is well understood when the problem is invariant under a non-compact group. Sections 2 and 3 are devoted to problems on $\mathbf{R}^{N}$. In this case, the problem is invariant under translations. Sections 4 and 5 are devoted to critical exponents. The problem is then invariant under dilations. We try to emphazise the similarities between the two cases. In sections 2 and 4, problems are solved because of their symmetry. In sections 3 and 5, problems are solved by a symmetry breaking. Although the results are known, the proofs, specially of theorem 4.4, are simpler.

We will use the following functional spaces.

Definition 1.1. The space

$$
H^{1}\left(\mathbf{R}^{N}\right):=\left\{u \in L^{2}\left(\mathbf{R}^{N}\right): \nabla u \in L^{2}\left(\mathbf{R}^{N}\right)\right\}
$$

with the inner product

$$
(u, v)_{1}:=\int_{\mathbf{R}^{N}}[\nabla u \cdot \nabla v+u v]
$$

and the corresponding norm

$$
\|u\|_{1}:=\left(\int_{\mathbf{R}^{N}}|\nabla u|^{2}+|u|^{2}\right)^{1 / 2}
$$

is a Hilbert space. Let $\Omega$ be an open subset of $\mathbf{R}^{N}$. The space $H_{0}^{1}(\Omega)$ is the closure of $\mathcal{D}(\Omega)$ in $H^{1}\left(\mathbf{R}^{N}\right)$.

1991 Mathematics Subject Classification: Primary 58E35; Secondary 49J45.

The paper is in final form and no version of it will be published elsewhere. 
Let $N \geq 3$ and $2^{*}:=2 N /(N-2)$. The space

$$
\mathcal{D}^{1,2}\left(\mathbf{R}^{N}\right):=\left\{u \in L^{2^{*}}\left(\mathbf{R}^{N}\right): \nabla u \in L^{2}\left(\mathbf{R}^{N}\right)\right\}
$$

with the inner product

and the corresponding norm

$$
\int_{\mathbf{R}^{N}} \nabla u \cdot \nabla v
$$

$$
\left(\int_{\mathbf{R}^{N}}|\nabla u|^{2}\right)^{1 / 2}
$$

is a Hilbert space. The space $\mathcal{D}_{0}^{1,2}(\Omega)$ is the closure of $\mathcal{D}(\Omega)$ in $\mathcal{D}^{1,2}\left(\mathbf{R}^{N}\right)$. For the simplicity of notations, we shall write $2^{*}=\infty$ when $N=1$ or $N=2$.

For the following results, see [3] or [9].

THEOREM 1.2. (Sobolev imbedding theorem). The following embeddings are continuous:

$$
\begin{array}{ll}
H^{1}\left(\mathbf{R}^{N}\right) \subset L^{p}\left(\mathbf{R}^{N}\right), & 2 \leq p<\infty, N=1,2, \\
H^{1}\left(\mathbf{R}^{N}\right) \subset L^{p}\left(\mathbf{R}^{N}\right), & 2 \leq p \leq 2^{*}, N \geq 3, \\
\mathcal{D}^{1,2}\left(\mathbf{R}^{N}\right) \subset L^{2^{*}}\left(\mathbf{R}^{N}\right), & N \geq 3 .
\end{array}
$$

In particular, the Sobolev inequality holds:

$$
S:=\inf _{\substack{u \in \mathcal{D}^{1,2}\left(\mathbf{R}^{N}\right) \\|u|_{2^{*}=1}}}|\nabla u|_{2}^{2}>0 .
$$

ThEOREm 1.3. (Rellich imbedding theorem). If $|\Omega|<\infty$, the following embeddings are compact:

$$
H_{0}^{1}(\Omega) \subset L^{p}(\Omega), \quad 2 \leq p<2^{*} .
$$

Corollary 1.4. (Poincaré inequality). If $|\Omega|<\infty$, then

$$
\lambda_{1}(\Omega):=\inf _{\substack{u \in H_{0}^{1}(\Omega) \\|u|_{2}=1}}|\nabla u|_{2}^{2}>0
$$

is achieved.

Remarks 1.5. a) It is clear that $H_{0}^{1}(\Omega) \subset \mathcal{D}_{0}^{1,2}(\Omega)$.

b) If $|\Omega|<\infty$, Poincaré inequality implies that $H_{0}^{1}(\Omega)=\mathcal{D}_{0}^{1,2}(\Omega)$.

2. Subcritical Sobolev inequalities. Let $N \geq 2$ and $2<p<2^{*}$. Sobolev theorem implies that

$$
S_{p}:=\inf _{\substack{u \in H^{1}\left(\mathbf{R}^{N}\right) \\|u|_{p}=1}}\|u\|_{1}^{2}>0 .
$$

In order to prove that the infimum is achieved, we consider a minimizing sequence $\left(u_{n}\right) \subset H^{1}\left(\mathbf{R}^{N}\right):$

$$
\left|u_{n}\right|_{p}=1, \quad\left\|u_{n}\right\|_{1}^{2} \rightarrow S_{p}, \quad n \rightarrow \infty .
$$

Going if necessary to a subsequence, we may assume $u_{n} \rightarrow u$ in $H^{1}\left(\mathbf{R}^{N}\right)$, so that

$$
\|u\|_{1}^{2} \leq \underline{\lim }\left\|u_{n}\right\|_{1}^{2}=S_{p} .
$$


Thus $u$ is a minimizer provided $|u|_{p}=1$. But we know only that $|u|_{p} \leq 1$. Indeed, for any $v \in H^{1}$ and $y \in \mathbf{R}^{N}$ the translated function

$$
v^{y}(x):=v(x+y)
$$

satisfies

$$
\left\|v^{y}\right\|_{1}=\|v\|_{1}, \quad\left|v^{y}\right|_{p}=|v|_{p} .
$$

Hence the problem is invariant by the noncompact group of translations. In order to overcome this difficulty, we will use the following result.

LEMMA 2.1. (Brezis-Lieb, 1983). Let $\Omega$ be an open subset of $\mathbf{R}^{N}$ and let $\left(u_{n}\right) \subset$ $L^{p}(\Omega), 1 \leq p<\infty$. If

a) $\left(u_{n}\right)$ is bounded in $L^{p}(\Omega)$,

b) $u_{n} \rightarrow u$ almost everywhere on $\Omega$, then

$$
\lim _{n \rightarrow \infty}\left(\left|u_{n}\right|_{p}^{p}-\left|u_{n}-u\right|_{p}^{p}\right)=|u|_{p}^{p} .
$$

Proof. See [4], [9] or [10].

Remarks 2.2. a) The preceding lemma is a refinement of Fatou's lemma.

b) Under the assumptions of the lemma, $u_{n} \rightarrow u$ weakly in $L^{p}(\Omega)$. However, weak convergence in $L^{p}(\Omega)$ is not sufficient to obtain the conclusion, except when $p=2$.

c) In any Hilbert space

$$
u_{n} \rightarrow u \Rightarrow \lim _{n \rightarrow \infty}\left(\left|u_{n}\right|^{2}-\left|u_{n}-u\right|^{2}\right)=|u|^{2} .
$$

Lemma 2.3. Let $r>0$ and $2 \leq q<2^{*}$. If $\left(u_{n}\right)$ is bounded in $H^{1}\left(\mathbf{R}^{N}\right)$ and if

$$
\sup _{y \in \mathbf{R}^{N}} \int_{B(y, r)}\left|u_{n}\right|^{q} \rightarrow 0, n \rightarrow \infty
$$

then $u_{n} \rightarrow 0$ in $L^{p}\left(\mathbf{R}^{N}\right)$ for $2<p<2^{*}$.

Proof. See [7].

THEOREM 2.4. (P.L. Lions, 1984). Let $\left(u_{n}\right) \subset H^{1}\left(\mathbf{R}^{N}\right)$ be a minimizing sequence satisfying (1). Then there exists a sequence $\left(y_{n}\right) \subset \mathbf{R}^{N}$ such that $u_{n}^{y_{n}}$ contains a convergent subsequence. In particular there exists a minimizer for $S_{p}$.

Proof. Since $\left|u_{n}\right|_{p}=1$, lemma 2.3 implies that

$$
\delta:=\underline{\lim _{n \rightarrow \infty}} \sup _{y \in \mathbf{R}^{N}} \int_{B(y, r)}\left|u_{n}\right|^{2}>0 .
$$

Going if necessary to a subsequence, we may assume the existence of $\left(y_{n}\right) \subset \mathbf{R}^{N}$ such that

$$
\int_{B\left(y_{n}, r\right)}\left|u_{n}\right|^{2}>\delta / 2
$$

Let us define $v_{n}:=u_{n}^{y_{n}}$. Hence $\left|v_{n}\right|_{p}=1,\left\|v_{n}\right\|_{1}^{2} \rightarrow S_{p}$ and

$$
\int_{B(0, r)}\left|v_{n}\right|^{2}>\delta / 2
$$


Since $\left(v_{n}\right)$ is bounded in $H^{1}\left(\mathbf{R}^{N}\right)$, we may assume, going if necessary to a subsequence

$$
\begin{array}{ll}
v_{n} \rightarrow v & \text { in } H^{1}\left(\mathbf{R}^{N}\right), \\
v_{n} \rightarrow v & \text { in } L_{\mathrm{loc}}^{2}\left(\mathbf{R}^{N}\right), \\
v_{n} \rightarrow v & \text { a.e. on } \mathbf{R}^{N} .
\end{array}
$$

By Brezis-Lieb lemma,

$$
1=|v|_{p}^{p}+\lim \left|w_{n}\right|_{p}^{p}
$$

where $w_{n}:=v_{n}-v$. Hence we have

$$
\begin{aligned}
S_{p}=\lim \left\|v_{n}\right\|_{1}^{2} & =\|v\|_{1}^{2}+\lim \left\|w_{n}\right\|_{1}^{2} \\
& \geq S_{p}\left[\left(|v|_{p}^{p}\right)^{2 / p}+\left(1-|v|_{p}^{p}\right)^{2 / p}\right] .
\end{aligned}
$$

Since, by (2), $v \neq 0$, we obtain $|v|_{p}^{p}=1$, so that $\|v\|_{1}^{2}=S_{p}=\lim \left\|v_{n}\right\|_{1}^{2}$.

THEOREM 2.5. There exists a radially symmetric, positive, $\mathcal{C}^{2}$ minimizer for $S_{p}$.

Proof. 1) By the preceding theorem, there exists a minimizer $u \in H^{1}\left(\mathbf{R}^{N}\right)$ for $S_{p}$. Using symmetrization ([6]), we may assume that $u$ is radially symmetric. Replacing $u$ by $|u|$, we may also assume that $u$ is non-negative.

2 ) It follows from Lagrange multiplier rule ([9]) that, for some $\lambda>0, u$ is a solution of

$$
-\Delta u+u=\lambda u^{p-1} .
$$

By Brezis-Kato theorem, $u \in \mathcal{C}^{2}\left(\mathbf{R}^{N}\right)$. The strong maximum principle implies that $u$ is positive.

3. Subcritical problem. Motivated by a nonlinear Schrödinger equation, we consider the following minimization problem:

$$
S_{V}:=\inf _{\substack{u \in H^{1}\left(\mathbf{R}^{N}\right) \\|u|_{p}=1}} \int_{\mathbf{R}^{N}}\left[|\nabla u|^{2}+V(x) u^{2}\right] d x,
$$

where $N \geq 2$ and $2<p<2^{*}$. We assume that $V \in \mathcal{C}\left(\mathbf{R}^{N}\right)$ satisfies

$$
0<\inf _{x \in \mathbf{R}^{N}} V(x)<\sup _{x \in \mathbf{R}^{N}} V(x)=\lim _{|x| \rightarrow \infty} V(x)=1 .
$$

By scaling, it is easy to replace 1 by any positive number. On $H^{1}\left(\mathbf{R}^{N}\right)$, we define the equivalent norm

$$
\|u\|^{2}:=\int_{\mathbf{R}^{N}}\left[|\nabla u|^{2}+V(x) u^{2}\right] d x .
$$

We consider a minimizing sequence $\left(u_{n}\right) \subset H^{1}\left(\mathbf{R}^{N}\right)$ satisfying

$$
\left|u_{n}\right|_{p}=1, \quad\left\|u_{n}\right\|^{2} \rightarrow S_{V}, \quad n \rightarrow \infty .
$$

THEOREM 3.1. Let $\left(u_{n}\right) \subset H^{1}\left(\mathbf{R}^{N}\right)$ be a minimizing sequence satisfying (4). Under assumption $(3),\left(u_{n}\right)$ contains a convergent subsequence. In particular, there exists a minimizer for $S_{V}$.

Proof. 1) Let $u>0$ be a minimizer for $S_{p}$. Assumption (3) implies that

$$
S_{V} \leq\|v\|^{2}<\|v\|_{1}^{2}=S_{p}
$$


2) Since $\left(u_{n}\right)$ is bounded in $H^{1}\left(\mathbf{R}^{N}\right)$, we may assume, going if necessary to a subsequence,

Brezis-Lieb lemma leads to

$$
\begin{array}{ll}
u_{n} \rightarrow u & \text { in } H^{1}\left(\mathbf{R}^{N}\right), \\
u_{n} \rightarrow u & \text { in } L_{\mathrm{loc}}^{p}\left(\mathbf{R}^{N}\right), \\
u_{n} \rightarrow u & \text { a.e. on } \mathbf{R}^{N} .
\end{array}
$$

$$
1=|u|_{p}^{p}+\lim \left|w_{n}\right|_{p}^{p}
$$

where $w_{n}:=u_{n}-u$. Hence we have

$$
\begin{aligned}
S_{V}=\lim \left\|u_{n}\right\|^{2} & =\|u\|^{2}+\lim \left\|w_{n}\right\|^{2} \\
& =\|u\|^{2}+\lim \left\|w_{n}\right\|_{1}^{2} \\
& \geq S_{V}|u|_{p}^{2}+S_{p}\left(1-|u|_{p}^{p}\right)^{2 / p} .
\end{aligned}
$$

Since, by the first step, $S_{V}<S_{p}$, we obtain $|u|_{p}=1$, so that

$$
\|u\|^{2}=S_{V}=\lim \left\|u_{n}\right\|^{2} .
$$

4. Critical Sobolev inequality. Let $N \geq 3$. The optimal constant in Sobolev inequality is given by

$$
S:=\inf _{\substack{u \in \mathcal{D}^{1,2}\left(\mathbf{R}^{N}\right) \\|u|_{2^{*}=1}}}|\nabla u|_{2}^{2}>0 .
$$

In order to prove that the infimum is achieved, we consider a minimizing sequence $\left(u_{n}\right) \subset$ $\mathcal{D}^{1,2}\left(\mathbf{R}^{N}\right)$ :

$$
\left|u_{n}\right|_{2^{*}}=1,\left|\nabla u_{n}\right|_{2}^{2} \rightarrow S, n \rightarrow \infty
$$

Going if necessary to a subsequence, we may assume $u_{n} \rightarrow u$ in $\mathcal{D}^{1,2}\left(\mathbf{R}^{N}\right)$, so that

$$
|\nabla u|_{2}^{2} \leq \underline{\lim }\left|\nabla u_{n}\right|_{2}^{2}=S .
$$

Thus $u$ is a minimizer provided $|u|_{2^{*}}=1$. But we know only that $|u|_{2^{*}} \leq 1$. Indeed, for any $v \in \mathcal{D}^{1,2}, y \in \mathbf{R}^{N}$ and $\lambda>0$, the rescaled function

$$
v^{y, \lambda}(x):=\lambda^{(N-2) / 2} v(\lambda x+y)
$$

satisfies

$$
\left|\nabla v^{y, \lambda}\right|_{2}=|\nabla v|_{2}, \quad\left|v^{y, \lambda}\right|_{2^{*}}=|v|_{2^{*}} .
$$

Hence the problem is invariant by translations and dilations. In order to exclude noncompactness, we will use some results from measure theory (see [9]).

Definition 4.1. Let $\Omega$ be an open subset of $\mathbf{R}^{N}$ and define

$$
\begin{gathered}
\mathcal{K}(\Omega):=\{u \in \mathcal{C}(\Omega): \operatorname{supp} u \text { is a compact subset of } \Omega\}, \\
\mathcal{B C}(\Omega):=\left\{u \in \mathcal{C}(\Omega):|u|_{\infty}:=\sup _{x \in \Omega}|u(x)|<\infty\right\} .
\end{gathered}
$$

The space $\mathcal{C}_{0}(\Omega)$ is the closure of $\mathcal{K}(\Omega)$ in $\mathcal{B C}(\Omega)$ with respect to the uniform norm. A finite measure on $\Omega$ is a continuous linear functional on $\mathcal{C}_{0}(\Omega)$. The norm of the finite 
measure $\mu$ is defined by

$$
\|\mu\|:=\sup _{\substack{u \in \mathcal{C}_{0}(\Omega) \\|u|_{\infty}=1}}|\langle\mu, u\rangle|
$$

We denote by $\mathcal{M}(\Omega)$ (resp. $\mathcal{M}^{+}(\Omega)$ ) the space of finite measures (resp. positive finite measures) on $\Omega$. A sequence $\left(\mu_{n}\right)$ converges weakly to $\mu$ in $\mathcal{M}(\Omega)$, written

$$
\mu_{n} \rightarrow \mu,
$$

provided

$$
\left\langle\mu_{n}, u\right\rangle \rightarrow\langle\mu, u\rangle, \forall u \in \mathcal{C}_{0}(\Omega) .
$$

THEOREM 4.2. a) Every bounded sequence of finite measures on $\Omega$ contains a weakly convergent subsequence.

b) If $\mu_{n} \rightarrow \mu$ in $\mathcal{M}(\Omega)$ then $\left(\mu_{n}\right)$ is bounded and

$$
\|\mu\| \leq \underline{\lim }\left\|\mu_{n}\right\| .
$$

c) If $\mu \in \mathcal{M}^{+}(\Omega)$ then

$$
\|\mu\|=\langle\mu, 1\rangle=\sup _{\substack{u \in \mathcal{B C}(\Omega) \\|u|_{\infty}=1}}\langle\mu, u\rangle .
$$

Following P.L. Lions [8], Bianchi, Chabrowski, Szulkin [2] and Ben Naoum, Troestler, Willem [1], we describe the lack of compactness of the injection $\mathcal{D}^{1,2}\left(\mathbf{R}^{N}\right) \subset L^{2^{*}}\left(\mathbf{R}^{N}\right)$.

LEMmA 4.3. (Concentration-compactness lemma). Let $\left(u_{n}\right) \subset \mathcal{D}^{1,2}\left(\mathbf{R}^{N}\right)$ be a sequence such that

and define

$$
\begin{aligned}
u_{n} \rightarrow u, & \text { in } \mathcal{D}^{1,2}\left(\mathbf{R}^{N}\right), \\
\left|\nabla\left(u_{n}-u\right)\right|^{2} \rightarrow \mu, & \text { in } \mathcal{M}\left(\mathbf{R}^{N}\right), \\
\left|u_{n}-u\right|^{2^{*}} \rightarrow \nu, & \text { in } \mathcal{M}\left(\mathbf{R}^{N}\right), \\
u_{n} \rightarrow u, & \text { a.e. on } \mathbf{R}^{N}
\end{aligned}
$$

$$
\mu_{\infty}:=\lim _{R \rightarrow \infty} \varlimsup_{n \rightarrow \infty} \int_{|x| \geq R}\left|\nabla u_{n}\right|^{2}, \quad \nu_{\infty}:=\lim _{R \rightarrow \infty} \varlimsup_{n \rightarrow \infty} \int_{|x|>R}\left|u_{n}\right|^{2^{*}} .
$$

Then it follows that

$$
\begin{gathered}
\|\nu\|^{2 / 2^{*}} \leq S^{-1}|| \mu||, \\
\nu_{\infty}^{2 / 2^{*}} \leq S^{-1} \mu_{\infty}, \\
\varlimsup_{n \rightarrow \infty}\left|\nabla u_{n}\right|_{2}^{2}=|\nabla u|_{2}^{2}+\|\mu\|+\mu_{\infty}, \\
\varlimsup_{n \rightarrow \infty}\left|u_{n}\right|_{2^{*}}^{2^{*}}=|u|_{2^{*}}^{2^{*}}+\|\nu\|+\nu_{\infty} .
\end{gathered}
$$

Moreover, if $u=0$ and $\|\nu\|^{2 / 2^{*}}=S^{-1}\|\mu\|$, then $\nu$ is concentrated at a single point.

Pr o of. Inequality (6) is proved in [8] and inequality (7) in [2]. Equalities (8) and (9) are proved in [1]. (See also [9] and [10]). 
TheOREM 4.4. (P.L. Lions, 1985). Let $\left(u_{n}\right) \subset \mathcal{D}^{1,2}\left(\mathbf{R}^{N}\right)$ be a minimizing sequence satisfying (5). Then there exists a sequence $\left.\left(y_{n}, \lambda_{n}\right) \subset \mathbf{R}^{N} \times\right] 0, \infty\left[\right.$ such that $\left(u_{n}^{y_{n}, \lambda_{n}}\right)$ contains a convergent subsequence. In particular there exists a minimizer for $S$.

Pr o of. Define the Lévy concentration functions

$$
Q_{n}(\lambda):=\sup _{y \in \mathbf{R}^{N}} \int_{B(y, \lambda)}\left|u_{n}\right|^{2^{*}} .
$$

Since, for every $u$,

$$
\lim _{\lambda \rightarrow 0^{+}} Q_{n}(\lambda)=0, \quad \lim _{\lambda \rightarrow \infty} Q_{n}(\lambda)=1,
$$

there exists $\lambda_{n}>0$ such that $Q_{n}\left(\lambda_{n}\right)=1 / 2$. Moreover, there exists $y_{n} \in \mathbf{R}^{N}$ such that

$$
\int_{B\left(y_{n}, \lambda_{n}\right)}\left|u_{n}\right|^{2^{*}}=Q_{n}\left(\lambda_{n}\right)=1 / 2
$$

since

$$
\lim _{|y| \rightarrow \infty} \int_{B\left(y, \lambda_{n}\right)}\left|u_{n}\right|^{2^{*}}=0
$$

Let us define $v_{n}:=u_{n}^{y_{n}, \lambda_{n}}$. Hence $\left|v_{n}\right|_{2^{*}}=1,\left|\nabla v_{n}\right|_{2}^{2} \rightarrow S$ and

$$
\frac{1}{2}=\int_{B(0,1)}\left|v_{n}\right|^{2^{*}}=\sup _{y \in \mathbf{R}^{N}} \int_{B(y, 1)}\left|v_{n}\right|^{2^{*}} .
$$

Since $\left(v_{n}\right)$ is bounded in $\mathcal{D}^{1,2}\left(\mathbf{R}^{N}\right)$, we may assume, going if necessary to a subsequence,

$$
\begin{aligned}
v_{n} \rightarrow v, & \text { in } \mathcal{D}^{1,2}\left(\mathbf{R}^{N}\right), \\
\left|\nabla\left(v_{n}-v\right)\right|^{2} \rightarrow \mu, & \text { in } \mathcal{M}\left(\mathbf{R}^{N}\right), \\
\left|v_{n}-v\right|^{2^{*}} \rightarrow \nu, & \text { in } \mathcal{M}\left(\mathbf{R}^{N}\right), \\
v_{n} \rightarrow v, & \text { a.e. on } \mathbf{R}^{N} .
\end{aligned}
$$

By the preceding lemma,

$$
\begin{gathered}
S=\lim \left|\nabla v_{n}\right|_{2}^{2}=|\nabla v|_{2}^{2}+|| \mu||+\mu_{\infty}, \\
1=\left|v_{n}\right|_{2^{*}}^{2^{*}}=|v|_{2^{*}}^{2^{*}}+\| \nu||+\nu_{\infty},
\end{gathered}
$$

where

$$
\mu_{\infty}:=\lim _{R \rightarrow \infty} \varlimsup_{n \rightarrow \infty} \int_{|x|>R}\left|\nabla v_{n}\right|^{2}, \quad \nu_{\infty}:=\lim _{R \rightarrow \infty} \varlimsup_{n \rightarrow \infty} \int_{|x|>R}\left|v_{n}\right|^{2^{*}} .
$$

We deduce from (11), (6), (7) and Sobolev inequality,

$$
S \geq S\left(\left(|v|_{2^{*}}^{2^{*}}\right)^{2 / 2^{*}}+\| \nu||^{2 / 2^{*}}+\nu_{\infty}^{2 / 2^{*}}\right) .
$$

It follows from (12) that $|v|_{2^{*}}^{2^{*}},\|\nu\|$ and $\nu_{\infty}$ are equal either to 0 or to 1 . By (10), $\nu_{\infty} \leq 1 / 2$ so that $\nu_{\infty}=0$. If $\|\nu\|=1$ then $v=0$ and $\|\nu\|^{2 / 2^{*}} \geq S^{-1}\|\mu\|$. The preceding lemma implies that $\nu$ is concentrated at a single point $z$. We deduce from (10) the contradiction

$$
\frac{1}{2}=\sup _{y \in \mathbf{R}^{N}} \int_{B(y, 1)}\left|v_{n}\right|^{2^{*}} \geq \int_{B(z, 1)}\left|v_{n}\right|^{2^{*}} \rightarrow\|\nu\|=1 .
$$


Thus $|v|_{2^{*}}^{2^{*}}=1$ and so

$$
|\nabla v|_{2}^{2}=S=\lim \left|\nabla v_{n}\right|_{2}^{2} .
$$

Theorem 4.5. (Aubin, Talenti, 1976). The instanton

is a minimizer for $S$.

$$
U(x):=\frac{[N(N-2)]^{(N-2) / 4}}{\left[1+|x|^{2}\right]^{(N-2) / 2}}
$$

Proof. 1) By the preceding theorem, there exists a minimizer $u \in \mathcal{D}^{1,2}\left(\mathbf{R}^{N}\right)$ for $S$. Using symmetrization ([6]), we may assume that $u$ is radially symmetric. Replacing $u$ by $|u|$, we may also assume that $u$ is non-negative.

2) It follows from Lagrange multiplier rule ([9]) that, for some $\lambda>0, u$ is a solution of

$$
-\Delta u=\lambda u^{\frac{N+2}{N-2}} .
$$

By Brezis-Kato theorem, $u \in \mathcal{C}^{2}\left(\mathbf{R}^{N}\right)$. The strong maximum principle implies that $u$ is positive.

3) After scaling, we may assume

$$
-\Delta u=u^{\frac{N+2}{N-2}} .
$$

Moreover we can choose $\varepsilon>0$ such that

$$
U_{\varepsilon}(x):=\varepsilon^{(2-N) / 2} U(x / \varepsilon)
$$

satisfies

$$
U_{\varepsilon}(0)=u(0)
$$

But then $u$ and $U_{\varepsilon}$ are solutions of the problem

$$
\left\{\begin{array}{l}
\partial_{r}\left(r^{N-1} \partial_{r} v\right)=r^{N-1} v^{\frac{N+2}{N-2}}, r>0 \\
v(0)=u(0) \quad \partial_{r} v(0)=0
\end{array}\right.
$$

It follows easily that $u=U_{\varepsilon}$. By invariance, $U$ is a minimizer for $S$.

Proposition 4.6. For every open subset $\Omega$ of $\mathbf{R}^{N}$,

$$
S(\Omega):=\inf _{\substack{u \in \mathcal{D}^{1,2}(\Omega) \\|u|_{2^{*}=1}}}|\nabla u|_{2}^{2}=S
$$

and $S(\Omega)$ is never achieved except when $\Omega=\mathbf{R}^{N}$.

Proof. 1) It is clear that $S \leq S(\Omega)$. Let $\left(u_{n}\right) \subset \mathcal{D}\left(\mathbf{R}^{N}\right)$ be a minimizing sequence for $S$. We can choose $y_{n} \subset \mathbf{R}^{N}$ and $\lambda_{n}>0$ such that

$$
u_{n}^{y_{n}, \lambda_{n}} \in \mathcal{D}(\Omega) .
$$

Hence we obtain $S(\Omega) \leq S$.

2) Assume that $\Omega \neq \mathbf{R}^{N}$ and $u \in \mathcal{D}_{0}^{1,2}(\Omega)$ is a minimizer for $S(\Omega)$. By the preceding step, $u$ is also a minimizer for $S$. We may assume that $u \geq 0$, so that $u$ is a solution of

$$
-\Delta u=\lambda u^{\frac{N+2}{N-2}} .
$$

By the strong maximum principle, $u>0$ on $\mathbf{R}^{N}$. This is a contradiction, since $u \in$ $\mathcal{D}_{0}^{1,2}(\Omega)$. 
5. Critical exponents. This section is devoted to the Brezis-Nirenberg minimization problem

$$
S_{\lambda}:=\inf _{\substack{u \in H_{0}^{1}(\Omega) \\|u|_{2^{*}}=1}} \int_{\Omega}\left(|\nabla u|^{2}+\lambda u^{2}\right) d x
$$

where $N \geq 2, \Omega$ is a bounded open subset of $\mathbf{R}^{N}$ and $-\lambda_{1}(\Omega)<\lambda<0$. On $H_{0}^{1}(\Omega)$, we define the equivalent norm

$$
\|u\|^{2}:=\int_{\Omega}\left[|\nabla u|^{2}+\lambda u^{2}\right] d x .
$$

We consider a minimizing sequence $\left(u_{n}\right) \subset H_{0}^{1}(\Omega)$ satisfying

$$
\left|u_{n}\right|_{2^{*}}=1, \quad \|\left. u_{n}\right|^{2} \rightarrow S_{\lambda}, \quad n \rightarrow \infty .
$$

THEOREM 5.1. (Brezis-Nirenberg, 1983). Let $\left(u_{n}\right) \subset H_{0}^{1}(\Omega)$ be a minimizing sequence satisfying (13). If $N \geq 4$ and $-\lambda_{1}(\Omega)<\lambda<0$, then $\left(u_{n}\right)$ contains a convergent subsequence. In particular, there exists a minimizer for $S_{\lambda}$.

Proof. Since $\left(u_{n}\right)$ is bounded in $H_{0}^{1}(\Omega)$, we may assume, going if necessary to a subsequence,

$$
\begin{array}{ll}
u_{n} \rightarrow u & \text { in } H_{0}^{1}(\Omega), \\
u_{n} \rightarrow u & \text { in } L^{2}(\Omega), \\
u_{n} \rightarrow u & \text { a.e. on } \Omega .
\end{array}
$$

Brezis-Lieb lemma leads to

$$
1=|u|_{2^{*}}^{2^{*}}+\lim \left|w_{n}\right|_{2^{*}}^{2^{*}}
$$

where $w_{n}:=u_{n}-u$. Hence we obtain

$$
\begin{aligned}
S_{\lambda}=\lim \left\|u_{n}\right\|^{2} & =\|u\|^{2}+\lim \left\|w_{n}\right\|^{2} \\
& =\|u\|^{2}+\lim \left|\nabla w_{n}\right|_{2}^{2} \\
& \geq S_{\lambda}|u|_{2^{*}}^{2}+S\left(1-|u|_{2^{*}}^{2^{*}}\right)^{2 / 2^{*}} .
\end{aligned}
$$

Since, by the next lemma, $S_{\lambda}<S$, we obtain $|u|_{2^{*}}=1$, and so

$$
\|u\|^{2}=S_{\lambda}=\lim \left\|u_{n}\right\|^{2} .
$$

If $U$ is the instanton, we have, for $\lambda<0$,

$$
\frac{\|\left. U\right|^{2}}{|U|_{2^{*}}^{2}}=\frac{|\nabla U|_{2}^{2}+\lambda|U|_{2}^{2}}{|U|_{2^{*}}^{2}}<\frac{|\nabla U|_{2}^{2}}{|U|_{2^{*}}^{2}}=S .
$$

Since $U \notin H_{0}^{1}(\Omega)$, it is necessary to "concentrate" $U$ near a point of $\Omega$ after multiplication by a trunction function.

LEMMA 5.2. Under the assumption of theorem 5.1, there exists a nonnegative $v \in$ $H_{0}^{1}(\Omega) \backslash\{0\}$ such that

$$
\|v\|^{2} /|v|_{2^{*}}^{2}<S
$$


Proof. We may assume that $0 \in \Omega$. Let $\psi \in \mathcal{D}(\Omega)$ be a nonnegative function such that $\psi \equiv 1$ on $B(0, \rho), \rho>0$, and define, for $\varepsilon>0$,

$$
\begin{aligned}
& U_{\varepsilon}(x):=\varepsilon^{(2-N) / 2} U(x / \varepsilon), \\
& u_{\varepsilon}(x):=\psi(x) U_{\varepsilon}(x) .
\end{aligned}
$$

It follows from theorem 4.5 that

$$
\left|\nabla U_{\varepsilon}\right|_{2}^{2}=\left|U_{\varepsilon}\right|_{2^{*}}^{2^{*}}=S^{N / 2}
$$

As $\varepsilon \rightarrow 0^{+}$, we have that

$$
\begin{aligned}
\int_{\Omega}\left|\nabla u_{\varepsilon}\right|^{2} & =\int_{\mathbf{R}^{N}}\left|\nabla U_{\varepsilon}\right|^{2}+O\left(\varepsilon^{N-2}\right)=S^{N / 2}+O\left(\varepsilon^{N-2}\right), \\
\int_{\Omega}\left|u_{\varepsilon}\right|^{2^{*}} & =\int_{\mathbf{R}^{N}}\left|U_{\varepsilon}\right|^{2^{*}}+O\left(\varepsilon^{N}\right)=S^{N / 2}+O\left(\varepsilon^{N}\right), \\
\int_{\Omega}\left|u_{\varepsilon}\right|^{2} & =\int_{B(0, \rho)}\left|U_{\varepsilon}\right|^{2}+O\left(\varepsilon^{N-2}\right) \\
& \geq \int_{B(0, \varepsilon)} \frac{\left[N(N-2) \varepsilon^{2}\right]^{\frac{N-2}{2}}}{\left[2 \varepsilon^{2}\right]^{N-2}}+\int_{\varepsilon<|x|<\rho} \frac{\left[N(N-2) \varepsilon^{2}\right]^{\frac{N-2}{2}}}{\left[2|x|^{2}\right]^{N-2}}+O\left(\varepsilon^{N-2}\right) \\
& = \begin{cases}d \varepsilon^{2}|\ell n \varepsilon|+O\left(\varepsilon^{2}\right), & \text { if } N=4, \\
d \varepsilon^{2}+O\left(\varepsilon^{N-2}\right), & \text { if } N \geq 5,\end{cases}
\end{aligned}
$$

where $d$ is a positive constant. If $N=4$, we obtain

$$
\begin{aligned}
\frac{\left\|u_{\varepsilon}\right\|^{2}}{|u|_{2^{*}}^{2}} & \leq \frac{S^{2}+\lambda d \varepsilon^{2}|\ell n \varepsilon|+O\left(\varepsilon^{2}\right)}{\left(S^{2}+O\left(\varepsilon^{4}\right)\right)^{1 / 2}} \\
& =S+\lambda d \varepsilon^{2}|\ell n \varepsilon| S^{-1}+O\left(\varepsilon^{2}\right)<S,
\end{aligned}
$$

for $\varepsilon>0$ sufficiently small. And similarly, if $N \geq 5$, we have

$$
\begin{aligned}
\frac{\left\|u_{\varepsilon}\right\|^{2}}{\left|u_{\varepsilon}\right|_{2^{*}}^{2}} & \leq \frac{S^{N / 2}+\lambda d \varepsilon^{2}+O\left(\varepsilon^{N-2}\right)}{\left(S^{N / 2}+O\left(\varepsilon^{N}\right)\right)^{2 / 2^{*}}} \\
& =S+\lambda d \varepsilon^{2} S^{(2-N) / 2}+O\left(\varepsilon^{N-2}\right)<S,
\end{aligned}
$$

for $\varepsilon>0$ sufficiently small.

\section{References}

[1] A. K. Ben-Naoum, C. Troestler, M. Willem, Extrema problems with critical Sobolev exponents on unbounded domains, Nonlinear Analysis T.M.A. 26 (1996), 823-833.

[2] G. Bianchi, J. Chabrowski, A. Szulkin, On symmetric solutions of an elliptic equation with a nonlinearity involving critical Sobolev exponent, Nonlinear Analysis T.M.A. 25 (1995), 41-59.

[3] H. Brezis, Analyse fonctionnelle, Masson, Paris, 1983.

[4] H. Brezis and E. Lieb, A relation between pointwise convergence of functions and convergence of functionals, Proc. Amer. Math. Soc. 88 (1983), 486-490.

[5] H. Brezis and L. Nirenberg, Positive solutions of nonlinear elliptic equations involving critical Sobolev exponents, Comm. Pure Appl. Math. 36 (1983), 437-477. 
[6] E. Lieb, Existence and uniqueness of the minimizing solutions of Choquard's nonlinear equation, Stud. Appl. Math. 57 (1977), 93-105.

[7] P. L. Lions, The concentration-compactness principle in the calculus of variations. The locally compact case, Annales de l'Institut Henri Poincaré Analyse Non Linéaire 1 (1984) 105-145, 223-283.

[8] P. L. Lions, The concentration-compactness principle in the calculus of variations. The limit case, Revista Matematica Iberoamericana, 1 (1985) $\mathrm{N}^{\mathrm{O}} 1,145-201, \mathrm{~N}^{\mathrm{O}}$ 2, 45-120.

[9] M. Willem, Analyse harmonique réelle, Hermann, Paris, 1995.

[10] M. Willem, Minimax theorems, to appear. 